\title{
Impact of Virtual Synchronous Machines on Low-Frequency Oscillations in Power Systems
}

\author{
Muftau Baruwa and Meghdad Fazeli, Senior Member, IEEE
}

\begin{abstract}
The low-frequency oscillations (LFOs) inherent in power systems will be impacted by the increasing penetration of renewable energy sources (RESs). This paper investigates the impact of virtual synchronous machine (VSM) based RESs on the LFOs in power systems. A detailed two-machine test-bed has been developed to analyze the LFOs which exists when VSMs replace synchronous generators. The characteristics of the LFO modes and the dominant states have been comprehensively analyzed. Furthermore, this study analyzes the LFO modes which exists in an all-VSM grid. The role of the power system stabilizers in the all-VSM grid has been comprehensively evaluated. The IEEE benchmark two-area four-machine system has been employed to corroborate the results of the small-signal analysis and observe the transient performance. The analysis in this paper have been performed in MATLAB/SIMULINK environment.
\end{abstract}

Index Terms-Low-frequency oscillation, renewable energy sources, virtual synchronous machine, synchronous generator, power system stabilizer.

\section{INTRODUCTION}

$\mathbf{E}$ NVIRONMENTAL, technical and economic issues have been increasing the penetration of renewable energy sources (RESs) in the power system [1]. The replacement of synchronous generators (SGs) with large scale RESs has a significant impact on the power system dynamics and plays a crucial role on the system stability [2]-[5]. The British National Grid recently reported undesirable trends including increasing voltage and frequency oscillations, declining frequency response, inertia, reactive power support and short circuit level, which have been associated with high RESs penetration [6], [7]. For the UK to meet the 2050 target [8], without jeopardizing the grid stability and reliability, RESs must employ control paradigms which offer similar robustness as the conventional SGs. Consequently, several research has been done in the development and implementation of virtual synchronous machine (VSM) algorithms, which enable RESs mimic the behaviour of the SG, thus bolstering the grid stability and reliability. However, since VSMs emulate the dynamics of SGs, some of the undesirable characteristics of the SGs may also be exhibited by the VSM [9], [10]. Although several research have investigated the operation of the VSM

Manuscript received ...; revised ... and ...; accepted .... Date of publication ...; date of current version .... Paper no. ....

This work is supported by the FLEXIS project, which is part-funded by the European Regional Development Fund through the Welsh Government.

Muftau Baruwa is a PhD student with the Energy Safety Research Institute, College of Engineering, Swansea University, Swansea, SA1 8EN, U.K. (email: 750340@swansea.ac.uk).

Meghdad Fazeli is a Senior Lecturer with the Energy Safety Research Institute, College of Engineering, Swansea University, Swansea, SA1 8EN, U.K. (e-mail: m.fazeli@swansea.ac.uk). in a grid, only a handful of the previous arts [4], [9]-[16], have investigated the impact of the VSM on the low-frequency oscillations (LFOs) of power systems.

LFOs are an inherent phenomena in the conventional power system, and are usually in the range of $0.1-2 \mathrm{~Hz}$ [1], [3]. Although, LFOs generally refer to the electromechanical oscillations emanating from SGs, recent literature [17]-[21], have discussed the possibility of new modes in the LFO range due to the interaction between SGs and the RESs. Considering the critical nature of LFOs in impacting the power system stability and the gradual replacement of SGs by RESs, the threat of LFO instability will still exist. It is also noted that a "zero carbon power system" does not necessarily mean that there will not be any SGs. For examples, nuclear/hydro power plants, which are considered clean energy, utilize SGs. Hence in this paper, the oscillations in the LFO range emanating from both the RESs and SGs are termed as LFOs. Although, high frequency oscillations also occur in the interaction between RESs, the damping mechanisms on the virtual governor and virtual automatic voltage regulator (AVR) of the proposed VSM ensure adequate damping of high frequency oscillations [22].

One solution to mitigate the impact of LFOs, is to limit the magnitude of power transfer in critical tie-lines in order to reduce the stress in the system, however this results in the under-utilization of transmission line infrastructure [23], [24]. The modulation of demand side load has also been proposed in literature to damp LFOs [24]-[27]. The main idea is to remotely control end-user loads in response to LFOs. The loads are grouped into clusters and wide-area signals are utilized to control each clusters to achieve adequate damping of the LFOs. However, this technique requires the participation of significant end-user loads to achieve satisfactory performance, which may not be readily available at the instant of need. Moreover, the controllers need to be frequently reconfigured to capture changes in the load and network configuration [25]. An effective solution for damping LFOs is the deployment of power system stabilizers (PSSs) on SGs. This has been the prevalent and most economical technique for damping LFOs in power systems [4], [5]. The PSS injects supplementary stabilizing signals into the SG, thus mitigating the LFOs and improving the system stability [1], [28]. The rotor speed, frequency and power are commonly employed as input signals to the PSS [28]-[30]. However, the increasing replacement of SGs with RESs has posed new concerns and spurred several research works [11], [31], [32].

Although this study is mainly focused on the operation of RESs at the transmission level, similar trends may be 
expected for operation at the distribution level. Generally, two approaches have been employed in literature: (1) mitigating the impact of RESs on LFOs e.g. [33]-[36] (2) employing stabilizing signals on RESs to damp LFOs e.g. [37]-[40].

To mitigate the impact of RESs on LFOs, Sadamoto et al [33], proposed a retrofit controller for wind farms using a linear quadratic regulator that depends on partial feedback of states solely from the wind farm. This design ensures that newly added RESs do not destabilize a hitherto stable system. It was also observed in [17], [18], [34], [35], that optimizing the parameters of the phase-locked loop (PLL) reduced the impact of RESs on the damping of LFOs. Studies in [3], [36], [41] demonstrate that implementing voltage or reactive power control on RESs (as opposed to unity power factor control) relieves the reactive power strain on SGs and thus improves LFO damping. However, [20] claims that the interaction between reactive power control of RESs with the SGs can create new LFO modes. Also, changing the PLL's parameters as suggested in [17], [18], [34], [35] may impact the synchronization and the low-voltage ride-through performance. Moreover, since [33]-[36] emphasis on the mitigation of the detrimental impact of RESs on the power system, their employed parameter optimization, may not adequately damp external disturbances emanating from the SGs. Hence, the bulk of studies on RESs focus on the deployment of PSS and power oscillation dampers (PODs) using both local and wide-area signals to effectively damp LFOs [5], [37]-[40]. However, the potential frequency instability and inertia reduction associated with the traditional RESs is shifting the focus of new studies to the robust and grid-friendly VSMs [4]. Hence, this paper investigates the LFO modes which exists when VSMs replace SGs, and the role of PSS in such systems.

The concept of VSMs were originally introduced in [42], [43], where the voltage and phase angle of voltage source converters were modulated to mimic the dynamics of the SG. The algorithms in [42], [43] employed detailed models of the SG. However, recent studies have shown that these models are susceptible to numerical instability and are computationally intensive [44], [45]. Hence, subsequent VSM topologies proposed in literature have employed simpler control algorithms which emulate the SG dynamics on the power loop, voltage loop or current loop [45]-[47].

Recent studies [11], [13] have shown that LFOs, similar to the conventional SGs, exists for multi-VSM power systems. $\mathrm{Du}$ et al [11] observed that when the LFOs from the VSM and the SGs are in close proximity, the system angular stability deteriorates. Hence, the authors suggested designing the VSM parameters such that the LFOs from the VSM are not within close proximity of the LFOs from the SGs. Although this approach mitigates the detrimental impact of the VSM on the power system angular stability, it may lead to sub-optimal design and performance of the VSM. Alipoor et al [10] proposed a variable inertia scheme for damping LFOs. The rationale of this scheme was elucidated via the energy function analysis and validated via simulation. It was also reported that VSMs employing this scheme can be deployed as a buffer between the SG and the grid to enhance the system stability. However, the authors did not detail the stable boundaries for the inertia variation. Ref. [12] proposed augmenting the adaptive inertia with an inertia matching scheme. The change of frequency and the time derivative of the frequency change were utilized in deriving a stable threshold for the virtual inertia variation. However, the effectiveness of this scheme was not validated for large disturbances. In [9], the VSM swing equation was linearized and expressed as a second-order control system. Hence, the system parameters relating to the damping ratio can be optimized to improve the system stability. Results from the simulated scenarios prove that the proposed scheme is viable for damping LFOs. However, the design requires some trial and error procedures followed by complex and time consuming mathematical derivation. Although the above schemes for VSM do not employ an additional stabilizing signal, the damping provided by [10]-[12] is not as efficient as the conventional PSS.

Sun et al [13] proposed a lead-lag compensator employed on the frequency control loop, which provides both inertial and damping effect on the LFOs. Although the proposed control seems effective, the impact of the VSM replacing SG was not investigated. Huang et al [4] employed the damping torque analysis to evaluate the controllers which impact the LFO damping when VSMs replace SGs. The resulting analysis, illustrated that the PLL significantly impacts the LFO damping of the VSM. Hence, the authors proposed employing a lead-lag compensator which is cascaded with the PLL to improve the LFO damping of the VSM. However, the design of the proposed controller necessitates a tradeoff between the tracking speed of the PLL and the damping capability of the VSM, thus hindering the optimal damping of LFOs. Perez et al [14] investigated two LFO damping topologies namely: the grid-damping and self-damping, which employed the measured grid frequency and the virtual rotor speed of the VSM respectively. It was observed that the grid-damping is a more effective solution. Furthermore, the combination of both damping topologies provided satisfactory performance. Similarly, in [15], the difference between the measured grid frequency and the virtual rotor speed was synthesized to achieve a virtual inertial power to damp LFOs. However, the stabilizing signals for these topologies [14], [15] were injected on the active power loop, which may lead to torsional oscillations when the prime mover of the VSM is a wind turbine [1]. In [16], a virtual frictional component was proposed to damp LFOs. The input signal to the virtual frictional component is the difference in rotor speeds of the participating machines. Despite the efficacy of the proposed technique, it is highly reliant on the communication of remote signals (rotor speeds of remote machines), which institutes a technical and economical barrier for application in large systems [48].

In most of the previous arts (including [5], [13]), the grid network is assumed to be quasi-static, which is often considered adequate for stability analysis involving only SGs with slow dynamics [28], [29]. However, for RESs with fast response, evaluating the dynamic interaction of the RESs with the network is very crucial in validating the system stability [49], [50]. Also, in [9], [10], [13], [14] the analytical derivations do not provide a holistic insight of the system 


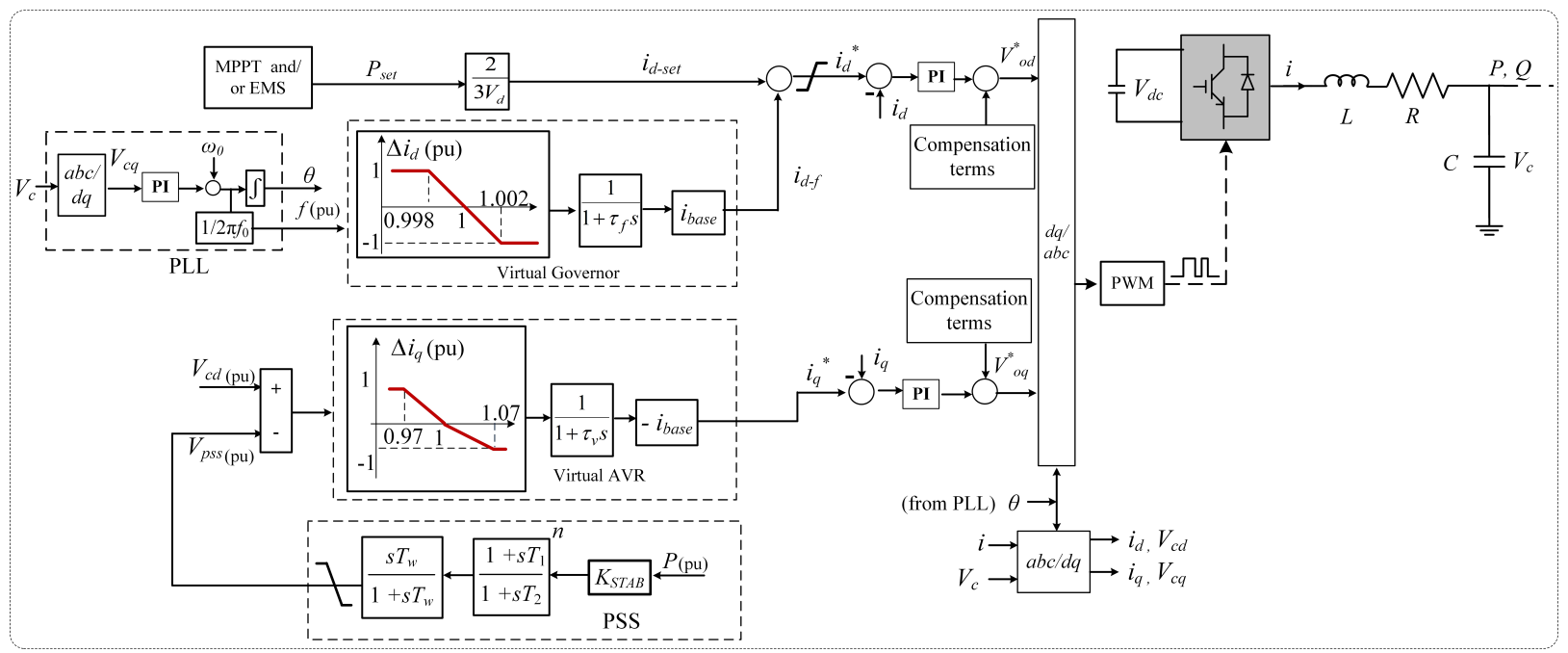

Fig. 1. Proposed VSMPSS control structure

stability as it neglects some controller dynamics (e.g. reactive power, voltage and current control). It is noted, that despite the vast works on RESs, there are still conflicting conclusions on its net impact on the LFO damping [32]. Furthermore, none of the previous arts (to the best of the authors knowledge) have evaluated the role of PSS in an all VSMs system. In light of the gaps in the previous works, the contributions of this paper are:

(1) Comprehensive analysis on the impact of replacing SG

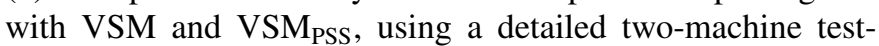
bed.

(2) Comprehensive analysis of the LFO modes which exists in an all-VSM power system.

(3) Evaluation of the role of PSS in an all-VSM power system.

It is noted that at the time of writing this paper there is no worldwide accepted definition on VSM topology, nor on its technical specifications. For example [51], that uses a direct voltage controlled approach, claims that although " $d q$-axis current injection" converters, which use a "Swing Equation-based inertial response", can provide a "fast frequency response", they do not have "the same nature as inertia naturally provided by the SG" and can "destabilize the system even more" claims, which are contradictory with [52]-[54]. The authors, therefore, recognize that the proposed structure in this paper may not be considered by some researchers as a typical VSM technology.

This paper is organized as follows: The modelling of the VSM is presented in Section II. Section III presents the comprehensive modal analysis of the power system using a detailed two-machine test-bed. Section IV presents the simulation results which corroborate the analysis in III. Section $\mathrm{V}$ concludes the paper.

\section{Modelling OF THE VSM}

VSMs are proposed to enable RESs behave like SGs. The base of the VSM method employed in this paper was developed in [22], for wind turbine applications. The main addition here is the PSS for damping LFOs. The salient feature of this VSM paradigm is that no switching operation is required in all modes of operation i.e. grid connected operation, islanding and low-voltage ride-through. The VSM control structure, which is illustrated in Fig. 1, employs the standard $d q$ frame current controllers.

\section{A. Virtual governor}

The primary function of the virtual governor is to regulate frequency $f$ within nominal value. This is achieved by regulating $f$ in proportion to the current demand $i_{d}$ (which is associated with the active power $P$ ). The equation describing the governor dynamics is represented in (1) and illustrated in Fig. 1 [22].

$$
i_{d}^{*}=K_{f}\left(f^{*}-f\right)\left(\frac{1}{1+\tau_{f} s}\right)+i_{d-s e t}
$$

Where $f^{*}, i_{d}^{*}$ and $i_{d-s e t}$ are the reference frequency, reference active current and equivalent current set point respectively. From (1) and Fig. 1, it is observed that the PLL, which provides $f$ for the virtual governor operates in both islanded and grid-connected modes, thus ensuring $f$ is well regulated in all operating modes. $P_{s e t}$, which determines $i_{d-s e t}$, can be derived from a maximum power point tracking (MPPT) algorithm (e.g. for wind systems in [22]). $K_{f}$ and $\tau_{f}$ are the droop gains and the damping filter time constant of the virtual governor respectively. $K_{f}$ is chosen such that $f$ is within the nominal range i.e. $0.99 \mathrm{pu}<f<1.01 \mathrm{pu}$ [55]. The PLL maintains the $d q$ frame alignment of the voltage $V_{c}$ i.e. $V_{c q}=$ 0 (hence $V_{c d} \approx\left|V_{c}\right|$ ).

\section{B. Virtual AVR}

The role of the virtual AVR, is to regulate $V_{c d}$ in proportion to the current demand $i_{q}$ (which is associated with the reactive power $Q$ ). The virtual AVR configuration is illustrated in Fig. 1 and described in (2), where $V_{d}^{*}, i_{q}^{*}, K_{v}$ and $\tau_{v}$ represent the reference voltage, reference reactive current, droop gains and damping filter time constant respectively [22]. $K_{v}$ is chosen such that $V_{c}$ is within the nominal range i.e. $0.9 \mathrm{pu}<V_{c}<1.1$ 
pu [55]. From (2) and Fig. 1, it is observed that the virtual AVR actively regulates $V_{c d}$ for all operating modes (grid-connected and islanded) and ensures fast-fault current injection during fault.

$$
i_{q}^{*}=-K_{v}\left(V_{c d}^{*}-V_{c d}\right)\left(\frac{1}{1+\tau_{v} s}\right)
$$

\section{Power System Stabilizer}

The primary function of the PSS is to damp LFOs, thus improving the stability of the power system [28]. The PSS is integrated to the virtual AVR as illustrated in Fig. 1. To achieve adequate damping on the system, the input signal of the PSS should contain sufficient information of the system oscillation [37], [38]. Here, we implement the $P$ signal, which is a function of the rotor angle difference $\Delta \delta$ between interconnected machines (for inductive grid) [28], [48]. This enables the use of a local variable $(P)$ with adequate information on remote signals $(\Delta \delta)$. The stabilizer gain $K_{S T A B}$ determines the amount of damping introduced by the PSS [28]. The phase compensation of the damping signal is provided by the leadlag filter, where $n$ represents the number of cascaded leadlag filters [29]. The saturation block limits the output of the PSS $V_{p s s}$, so it does not adversely impact the virtual AVR response [28]. The signal washout block serves as a high-pass filter, which passes all the signals in the frequency range of interest and eliminates steady state signals from $V_{p s s}$. The time constant $T_{w}$ of the washout block is normally in the range of 1 to 20 seconds for most applications [28]. The overall equation representing the integration of the virtual AVR with the PSS is given by (3):

$$
i_{q}^{*}=-K_{v}\left(V_{d}^{*}-\left(V_{d}-V_{p s s}\right)\right)\left(\frac{1}{1+\tau_{v} s}\right)
$$

\section{Small-Signal Stability Analysis}

The primary concern in the small-signal stability study of power systems is the nature of the angular oscillations, when the system is subjected to small disturbances [28]. The angular oscillations of interest are the LFOs [1], [3]. A single machine infinite bus model is commonly used to observe the stability and performance of SGs [28], [29]. However, this may not be adequate for systems containing diverse RESs, as it is unable to elucidate the interaction between the various power generating sources in the network. Hence in this study, a detailed two-machine test-bed has been developed which offers an excellent platform for the observation and comprehensive analyses of the interaction of modes between the SG and VSM. The topology of the test-bed is illustrated in Fig. 2, where $S_{L 1}$ and $S_{L 2}$ represent the apparent power demand by the load at area 1 and 2 respectively, while $S_{\text {tie }}$ represents the power transmitted through the interconnecting tie-line. $V_{d q}$ and $I_{d q}$ represent the $d q$ component of the voltage and current of the machines respectively. The subscript 1 and 2 denote variables relating to machine 1 and 2 respectively. The 6th-order model of the SG has been implemented in this study [50], [56]. In order to investigate the small-signal stability analysis, all units in the system must be synchronized to a common reference frame [28], [29]. The rotating frame of an arbitrary machine is

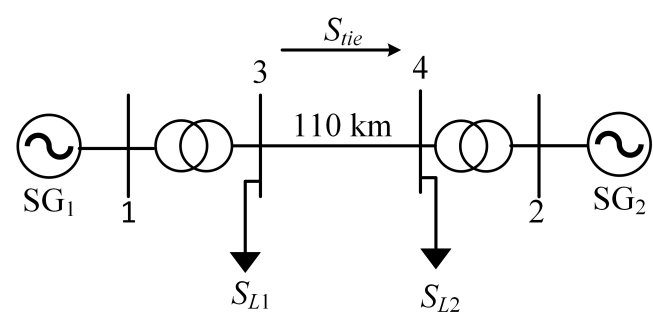

(a)

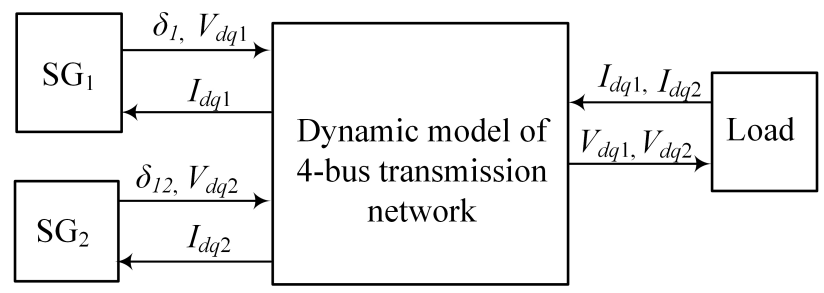

(b)

Fig. 2. Topology of two-machine test-bed: (a) Single line diagram (b) Signal-flow diagram

chosen as the common reference frame $D Q_{j}$ [28]. The output variables of the $i$ th machine rotating at $d q_{i}$ is translated to $D Q_{j}$ using the output transformation operator $T_{\text {out }}(5)$, while the input variables on $D Q_{j}$ are translated to $d q_{i}$ using $\left[T_{\text {out }}^{-1}\right]$ [49], [50].

$$
\begin{aligned}
& {\left[f_{D Q}\right]=\left[T_{\text {out }}\right]\left[f_{d q}\right]} \\
& {\left[T_{\text {out }}\right]=\left[\begin{array}{ll}
\cos \left(\delta_{i j}\right) & -\sin \left(\delta_{i j}\right) \\
\sin \left(\delta_{i j}\right) & \cos \left(\delta_{i j}\right)
\end{array}\right]}
\end{aligned}
$$

Where $f_{D Q}$ and $f_{d q}$ are the machine variables represented on $D Q_{j}$ and $d q_{i}$ respectively, $\delta_{i j}$ is the angular difference between $D Q_{j}$ and $d q_{i}$. The output and input of each machine are the machine voltage and current respectively. The network state equations and the loads are modelled in state space as detailed in [49], [56], and are all represented on the common reference frame. The complete state space model of the test system is modelled in MATLAB/SIMULINK. The linearized model of the complete system is represented by $\Delta \dot{x}=A \Delta x$ $+B \Delta u$ and is obtained in MATLAB using the linear analysis toolbox.

The stability analysis in this study analyzes the: (A) impact of SG replacement by VSM (B) robustness of the VSMPSS (C) stability of an all-VSM grid (D) the role of PSS in these scenarios. The system's parameters are detailed in Table I. The machine and network parameters have a base rating of 900 MVA. The nominal voltage at the SG terminal and transmission network are $20 \mathrm{kV}$ and $230 \mathrm{kV}$ respectively. The initial operating point for the machines are $P_{\text {set }}=P^{*}=0.8$ $\mathrm{pu}$.

\section{A. Impact of SG replacement by VSM}

The objective here is to observe the LFO modes when SGs are replaced by VSMs. To achieve this, we consider 
TABLE I

System's parameters

\begin{tabular}{|lll|}
\hline \multicolumn{3}{|c|}{ VSM } \\
\hline Parameter & Value \\
\hline Current Loop PI control & $K_{p}=9 \mathrm{e}-4$ & $K_{i}=9 \mathrm{e}-3$ \\
\hline$t_{f}, t_{v}$ & $1.3 \mathrm{~s}$, & $0.005 \mathrm{~s}$ \\
\hline PLL PI control & $K_{p}=0.005$ & $K_{i}=0.05$ \\
\hline Filter impedance & $R=0.008 \mathrm{pu}$ & $L=0.3 \mathrm{pu}$ \\
\hline \hline \multicolumn{2}{|c|}{$\mathrm{SG}$} \\
\hline Parameter & Value $(\mathrm{pu})$ \\
\hline$H, p$ & 6,2 \\
\hline$D$ & 0.002 \\
\hline \multicolumn{2}{|c|}{ Transmission network } \\
\hline \multicolumn{2}{|c|}{ Line impedance } & $R=9 \mathrm{e}-4 \mathrm{pu} / \mathrm{km}, X_{L}=9 \mathrm{e}-3 \mathrm{pu} / \mathrm{km}$ \\
\hline Transformer impedance & $X_{L}=0.15 \mathrm{pu}$ \\
\hline \multicolumn{2}{|c|}{$S_{L 1}, S_{L 2}, S_{\text {tie }}$} \\
\hline \multicolumn{2}{|c|}{$0.4 \mathrm{pu}, 0.8 \mathrm{pu}, 0.36 \mathrm{pu}(\mathrm{at} 0.85 \mathrm{PF})$} \\
\hline
\end{tabular}

three system configurations on the test-bed (see Fig. 2): (1) The stability of the test system with two SGs (SG-SG) (2) Replacing $\mathrm{SG}_{2}$ with VSM (SG-VSM) (3) Addition of PSS to

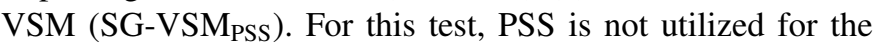
SG as this has been well established in literature [28], [29]. Fig. 3 illustrates the location of the dominant system poles for the three test configurations. Table II illustrates the damping ratio $(\zeta)$ and dominant states affecting the LFO modes. The dominant states are obtained using participation factor matrix $\left(p_{k}\right)$ [28]. The subscript 1 and 2 in the last column of Table II denote the dominant states relating to machine 1 and 2 respectively.

1) $S G-S G$ : For the first configuration, (see Fig. 3(a)). It is observed that, with 2 SGs (with no PSS) the system is marginally stable as a pair of poles oscillating at $5.52 \mathrm{rad} / \mathrm{s}$ are situated very close to the $j \omega$-axis. From Table II, it is observed that this poles are rotor angle modes (i.e. dominated by $\Delta \delta_{12}, \Delta \omega_{1}$ and $\left.\Delta \omega_{2}\right)$ and $\zeta=0.003$. This implies that a disturbance in the system will lead to continuous angular oscillations which do not decay with time, due to insufficient damping torque.

2) SG-VSM: With $\mathrm{SG}_{2}$ replaced by the VSM (see Fig. 3(a)), two oscillatory poles $\lambda_{1,2}$ and $\lambda_{3,4}$ are observed. The poles $\lambda_{1,2}$ oscillates at $2.62 \mathrm{rad} / \mathrm{s}$ and is dominated by $\Delta \delta_{12}$, $\Delta \omega_{1}$ and $\Delta i_{c d 2}^{*}$ (from the VSM's virtual governor). While $\lambda_{3,4}$ oscillates at $4.44 \mathrm{rad} / \mathrm{s}$, and is dominated by $\Delta \delta_{12}, \Delta i_{c d 2}^{*}$ and $\Delta \omega_{P L L 2}$ (from the VSM's PLL). This system is stable as the poles are all located on the left hand side (LHS) of the $j \omega$-axis. It is also noted that the damping ratio of $\lambda_{1,2}$ has increased. However, it is still not very well-damped $(\zeta$ $=0.092)$. It is noted that, although [11] suggests retuning the VSM's parameters, retuning an already well-designed VSM, may exacerbate other modes and deteriorate the VSM performance. As an alternative, since the VSM is designed to emulate the SG, some of the supplementary controls (e.g. PSS) employed on the SG, should be applicable on the VSM to enhance the power system stability.

3) $S G$-VSM $P S S$ : For this configuration, the PSS shown in Fig. 1 is employed on the VSM. From Fig. 3(b), it is observed that a direct feedback of $P$ (without lead-lag compensators) exacerbates the dominant LFO mode $\left(\lambda_{12}\right)$ in the SG-VSM configuration. Hence, to achieve the desired damping on the LFO modes, the PSS must be systematically designed. Two crucial parameters in the design of the PSS are the oscillatory frequency $\omega_{o s c}$ and the phase lead $\phi_{\text {lead }}$ on the lead-lag
TABLE II

Dominant eigenvalues for sub-section III-A

\begin{tabular}{|l|l|l|l|}
\hline \multicolumn{3}{|c|}{ SG-SG } \\
\hline Pole & Mode & Damping Ratio $(\zeta)$ & Dominant states \\
\hline$\lambda_{1,2}$ & $-0.017 \pm 5.52$ & 0.003 & $\Delta \delta_{12}, \Delta \omega_{1}, \Delta \omega_{2}$ \\
\hline \hline \multicolumn{3}{|c|}{ SG-VSM } \\
\hline Pole & Mode & Damping Ratio $(\zeta)$ & Dominant states \\
\hline$\lambda_{1,2}$ & $-0.242 \pm 2.62$ & 0.092 & $\Delta \delta_{12}, \Delta \omega_{1}, \Delta i_{c d 2}^{*}$ \\
\hline$\lambda_{3,4}$ & $-1.58 \pm 4.44$ & 0.335 & $\Delta \delta_{12}, \Delta \omega_{P L L 2}, \Delta i_{c d 2}^{*}$ \\
\hline \hline \multicolumn{3}{|c|}{ SG-VSM ${ }_{\text {PSS }}$} \\
\hline Pole & Mode & Damping Ratio $(\zeta)$ & Dominant states \\
\hline$\lambda_{1,2}$ & $-0.584 \pm 2.46$ & 0.23 & $\Delta \delta_{12}, \Delta \omega_{1}, \Delta i_{c d 2}^{*}, \Delta V_{p s s}$ \\
\hline$\lambda_{3,4}$ & $-4.3 \pm 4.62$ & 0.681 & $\Delta \omega_{P L L 2}, \Delta i_{c d 2}^{*}, \Delta V_{p s s}$ \\
\hline$\lambda_{5,6}$ & $-0.87 \pm 3.82$ & 0.209 & $\Delta \delta_{12}, \Delta \omega_{P L L 2}, \Delta V_{p s s}$ \\
\hline
\end{tabular}

compensator [29]. In this study, the required $\phi_{\text {lead }}$ is obtained using the residue approach [57], [58]. The residue angle $\angle R_{i}$ (see Fig. 3(b)), at which $\lambda_{12}$ approaches the $j \omega$-axis is obtained from the transfer function $G_{r e s}(s)=P / V^{*}$. Here $\angle R_{i} \approx 60^{\circ}$, hence the required $\phi_{\text {lead }}=120^{\circ}$ i.e. $\phi_{\text {lead }}=$ $180^{\circ}-\angle R_{i}$. The primary consideration in selecting $\omega_{o s c}$ for the PSS, is to achieve maximum damping on the critical modes. This is usually the inter-area modes which are usually under-damped due to weak interconnecting tie-lines [3], [5]. In this study, $\omega_{\text {osc }}$ is chosen as $4.21 \mathrm{rad} / \mathrm{s}$ which ensures adequate damping for the LFO modes of interest. It is noted that for complex PSS designs i.e. multi-band PSS (MB-PSS), more than one $\omega_{o s c}$ value is normally chosen within the LFO range [58]. For optimum performance, it is desirable to limit the maximum $\phi_{\text {lead }}$ of each compensator, such that $\phi_{\text {lead }}<$ $60^{\circ}$ [58]. Hence, three identical lead-lag compensators $(n=3)$ with $\phi_{\text {lead }}=40^{\circ}$ are implemented; such that $T_{1}$ and $T_{2}$ are 0.509 and 0.11 respectively. $K_{p s s}$ is chosen on the premise of achieving sufficient damping, whilst also avoiding controller saturation (which might occur due to very high $K_{p s s}$ ). Here, $K_{p s s}$ is chosen as 0.12 . To ensure fast response of the VSM, while passing the desired low frequencies, $T_{w}=1 \mathrm{~s}$.

From Fig. 3(c), it is observed that by employing the PSS, an additional oscillatory mode $\lambda_{5,6}$ is introduced into the system. This mode is dominated by $\Delta \delta_{12}, \Delta \omega_{P L L 2}$ and $\Delta V_{p s s}$. It is also observed that, the dominant modes in $\lambda_{1,2}$ and $\lambda_{3,4}$ are similar to configuration 2 . However, $\Delta V_{\text {pss }}$ is also dominant on $\lambda_{1,2}$ and $\lambda_{3,4}$. From Fig. 3(c), it is observed that the inclusion of the PSS moves the oscillatory modes further to the LHS, indicating improved stability and damping. As detailed in Table II, the $\zeta$ for $\lambda_{1,2}$ and $\lambda_{3,4}$ have increased to 0.23 and 0.681 respectively, which are now well-damped.

\section{B. Robustness of the VSM $M_{P S S}$}

Consider the testbed in Fig. 2, where $P_{1}$ and $P_{2}$ represent the active power generated by $\mathrm{SG}_{1}$ (or $\mathrm{VSM}_{1}$ ) and $\mathrm{SG}_{2}$ (or $\mathrm{VSM}_{2}$ ) respectively, and $P_{L}$ represents the aggregated active load (i.e. $P_{L 1}+P_{L 2}=P_{L}$ ). Since the network is inductive (i.e. $X_{L} \gg R$ ), one can assume that the SGs (or VSMs) rotor angles are small (i.e. $\sin \delta_{i} \approx \delta_{i}$ ), and the system voltage deviation is negligible (i.e. $V_{1}=V_{2} \approx 1 \mathrm{pu}$ ). The linear equation representing the active power flow in the network 


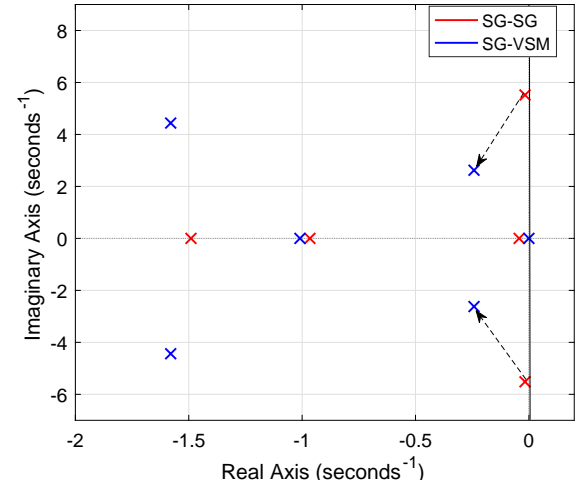

(a) Comparison of the LFO when VSM replaces SG

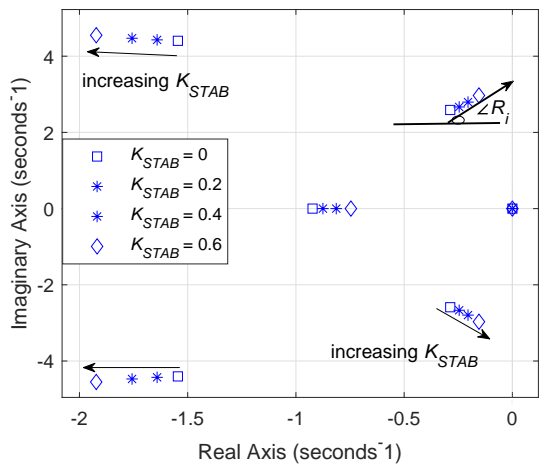

(b) Transition of LFO modes with direct feedback of $P$ without lead-lag compensators

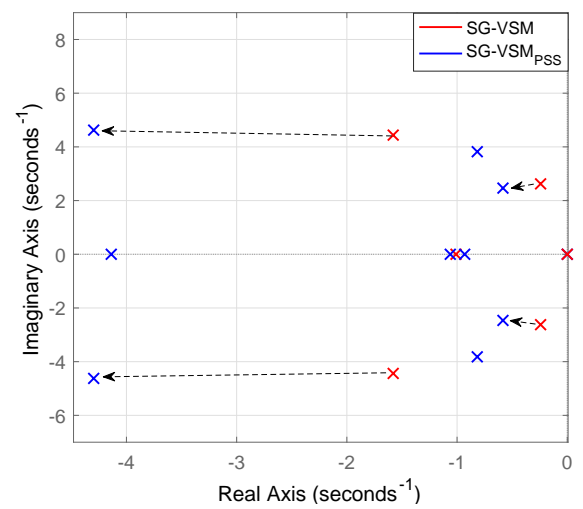

(c) Comparison of the LFO when PSS is employed on VSM

Fig. 3. Impact of SG replacement by VSM

can be represented by (6) [59]:

$$
\begin{aligned}
\Delta P_{1} & =\frac{1}{X_{L 1}}\left(\Delta \delta_{1}-\Delta \delta_{L}\right) \\
\Delta P_{2} & =\frac{1}{X_{L 2}}\left(\Delta \delta_{2}-\Delta \delta_{L}\right) \\
\Delta \delta_{L} & =\frac{\Delta \delta_{1} X_{L 2}+\Delta \delta_{2} X_{L 1}-\Delta P_{L} X_{L 1} X_{L 2}}{X_{L 1}+X_{L 2}}
\end{aligned}
$$

Where $\delta_{L}$ is the load angle, while $X_{L 1}$ and $X_{L 2}$ respectively represent the reactance of the transmission line connecting $\mathrm{SG}_{1}$ (or $\mathrm{VSM}_{1}$ ) and $\mathrm{SG}_{2}$ (or $\mathrm{VSM}_{2}$ ) to $P_{L}$. From (6), assuming that $\Delta \delta_{1}$ and $\Delta \delta_{2}$ are very small i.e. $\Delta \delta_{1}=\Delta \delta_{2} \approx 0, \Delta \delta_{L}$ can be represented as:

$$
\left|\Delta \delta_{L}\right| \approx \frac{\Delta P_{L} X_{L 1} X_{L 2}}{X_{L 1}+X_{L 2}}
$$

From (7), it is observed that variations in the loading condition $\Delta P_{L}$ and in the reactance of the transmission line $\Delta X_{L}$ are the main grid parameters that impact the small-signal (angular) stability of the system. It is noted that for a given transmission line, $X_{L}$ is proportional to the length of the transmission line.

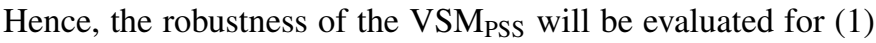
variations in the system loading, (2) variations in the length of the transmission tie-line $l_{t i e}$. The SG-VSMPSS setup in subsection III-A is employed here.

1) Impact of varying system loading: To observe the stability of the VSM $\mathrm{VSS}_{\mathrm{PS}}$ for varying load conditions, $S_{L 2}$ is varied from 0.6-1.2 pu. For the purpose of comparison, a scenario without PSS is also shown in the result. The Bode plot of the system frequency response is observed using the virtual governor loop, with $f^{*}$ and $f$ taken as the input and output of the open loop transfer function $G_{f}(s)$ respectively i.e. $G_{f}(s)=f / f^{*}$. From Fig. 4 , it is observed that without the PSS, although the phase margin (PM) is satisfactory i.e. $\mathrm{PM}=35^{\circ}$, the system has a relatively sharp peak at 2.6 $\mathrm{rad} / \mathrm{s}$ which indicates poor damping. With the $\mathrm{VSM}_{\mathrm{PSS}}$, the peak is eliminated for all scenarios indicating satisfactory damping. Furthermore, the PM is significantly improved for all configurations i.e. $93^{\circ} \leq \mathrm{PM} \leq 106^{\circ}$. The gain margin $(\mathrm{GM})$ for all scenarios is satisfactory, and is not impacted by the PSS i.e. $63 \mathrm{~dB} \leq \mathrm{GM} \leq 69 \mathrm{~dB}$.

2) Impact of variations in the length of the tie-line: The test here analyses the VSM $\mathrm{VSS}_{\mathrm{PS}}$ stability for different $l_{t i e}$, varying from $80-160 \mathrm{~km}$. The frequency response is also observed using $G_{f}(s)=f / f^{*}$. From Fig. 5, it is observed that without the PSS, although the PM is satisfactory (i.e. PM = 38 ${ }^{\circ}$ ) the system is not well damped at $\omega=2.6 \mathrm{rad} / \mathrm{s}$. With the $\mathrm{VSM}_{\mathrm{PSS}}$ employed, the system is well damped for all scenarios and the $\mathrm{PM}$ is improved i.e. $92^{\circ} \leq \mathrm{PM} \leq 103^{\circ}$. The $\mathrm{GM}$ for all scenarios is satisfactory, and is not impacted by the PSS i.e. $64 \mathrm{~dB} \leq \mathrm{GM} \leq 67 \mathrm{~dB}$.

\section{Stability analysis of an all-VSM grid}

The objectives here are to observe: (a) if the LFO modes observed in SG exists for an all-VSM grid (b) the states participating in the LFO (c) the impact of PSS on the overall system stability. To achieve this, two configurations are investigated (1) Both SGs in Fig. 2 are replaced by VSMs (VSM-VSM)

(2) PSS added on both VSMs (VSM PSS $-V_{S M S}$ ).

1) VSM-VSM: It is observed from Fig. 6 that the system is stable as all the modes are on the LHS of the $j \omega$-axis. Two modes $\lambda_{1,2}$ and $\lambda_{3,4}$ are observed oscillating at 4.44 $\mathrm{rad} / \mathrm{s}$ and $6.19 \mathrm{rad} / \mathrm{s}$ respectively. These modes are dominated by states from the PLL and governor. However, $\Delta \delta_{12}$ has minimal influence on $\lambda_{3,4}$. From Table III, it is observed that these modes are well damped. Moreover, comparing this configuration with SG-SG and SG-VSM (sub-section III-A), it is observed that the two modes $\left(\lambda_{1,2}\right.$ and $\left.\lambda_{3,4}\right)$ are much better damped in the VSM-VSM. 

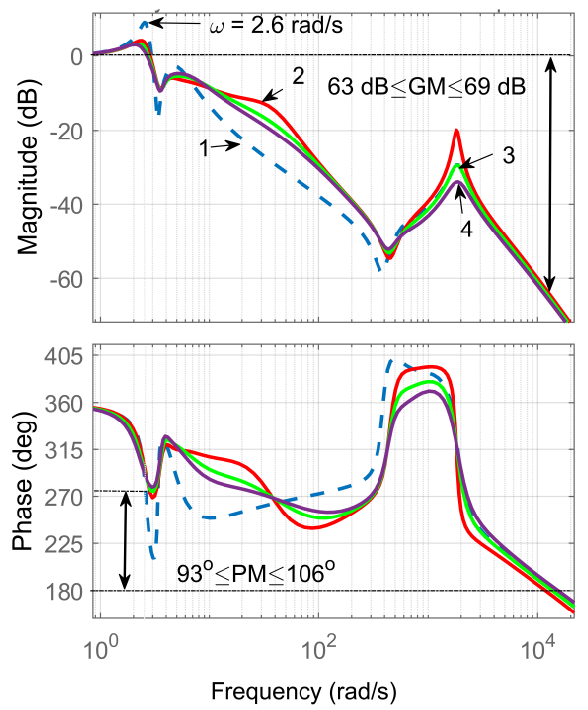

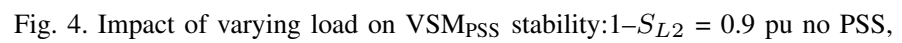
$2-S_{L 2}=0.6$ pu with PSS, $3-S_{L 2}=0.9 \mathrm{pu}$ with PSS, $4-S_{L 2}=1.2 \mathrm{pu}$ with PSS.
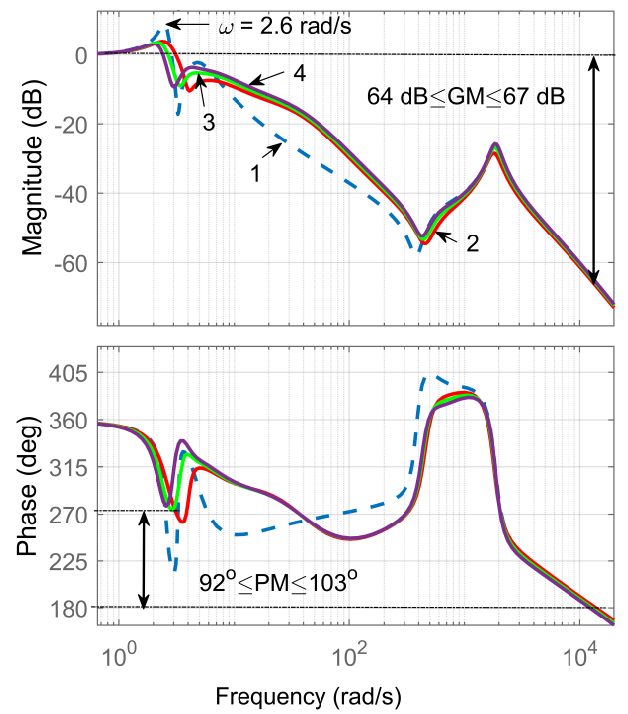

Fig. 5. Impact of varying length of tie-line on VSMPSS stability: $1-l_{t i e}=120$ $\mathrm{km}$ no PSS, $2-l_{t i e}=80 \mathrm{~km}$ with PSS, $3-l_{t i e}=120 \mathrm{~km}$ with PSS, $4-l_{t i e}=$ $160 \mathrm{~km}$ with PSS.

2) $V S M_{P S S}-V S M_{P S S}:$ With the inclusion of PSS, two additional modes $\lambda_{5,6}$ and $\lambda_{7,8}$ are introduced in the system. These modes are dominated by states from the PLL, virtual governor and PSS. It is observed from Fig. 6, that the PSS moves the modes further to the LHS indicating improved stability and damping (see Table III).

The observation from the small-signal analysis shows that, the replacement of SG with VSM improves the damping of interarea LFO. However, PSS is required for satisfactory operation. It is also observed that the $\mathrm{VSM}_{\mathrm{PSS}}$ is robust to variations in system operating condition. Furthermore, an all-VSM grid can operate satisfactorily without the need for a PSS, as the LFO
TABLE III

Dominant eigenvalues for sub-section III-B

\begin{tabular}{|l|l|l|l|}
\hline \multicolumn{3}{|c|}{ VSM-VSM } \\
\hline Pole & Mode & Damping & Dominant states \\
\hline$\lambda_{1,2}$ & $-1.22 \pm 4.44$ & 0.265 & $\begin{array}{l}\Delta \delta_{12}, \Delta \omega_{P L L 1}, \Delta \omega_{P L L 2}, i_{c d 1}^{*}, \\
i_{c d 2}^{*}\end{array}$ \\
\hline$\lambda_{3,4}$ & $-2.93 \pm 6.19$ & 0.428 & $\Delta \omega_{P L L 1}, \Delta \omega_{P L L 2}, i_{c d 1}^{*}, i_{c d 2}^{*}$ \\
\hline \hline \multicolumn{3}{|c|}{ VSM } & \multicolumn{3}{|c|}{ VSS- VSMPSS } \\
\hline Pole & Mode & Damping & Dominant states \\
\hline$\lambda_{1,2}$ & $-3.15 \pm 4.49$ & 0.575 & $\begin{array}{l}\Delta \delta_{12}, \Delta \omega_{P L L 1}, \Delta \omega_{P L L 2}, i_{c d 1}^{*}, \\
i_{c d 2}^{*}, \Delta V_{p s s}\end{array}$ \\
\hline$\lambda_{3,4}$ & $-5.75 \pm 5.33$ & 0.734 & $\begin{array}{l}\Delta \omega_{P L L 1}, \Delta \omega_{P L L 2}, i_{c d 1}^{*}, i_{c d 2}^{*}, \\
\Delta V_{p s s}\end{array}$ \\
\hline$\lambda_{5,6}$ & $-1.42 \pm 3.58$ & 0.368 & $\Delta \delta_{12}, \Delta \omega_{P L L 1}, \Delta \omega_{P L L 2}, i_{c d 1}^{*}$, \\
& & & $i_{c d 2}^{*}, \Delta V_{p s s}$ \\
\hline$\lambda_{7,8}$ & $-1.28 \pm 3.89$ & 0.3 & $\Delta \delta_{12}, \Delta \omega_{P L L 1}, \Delta \omega_{P L L 2}, \Delta V_{p s s}$ \\
\hline
\end{tabular}

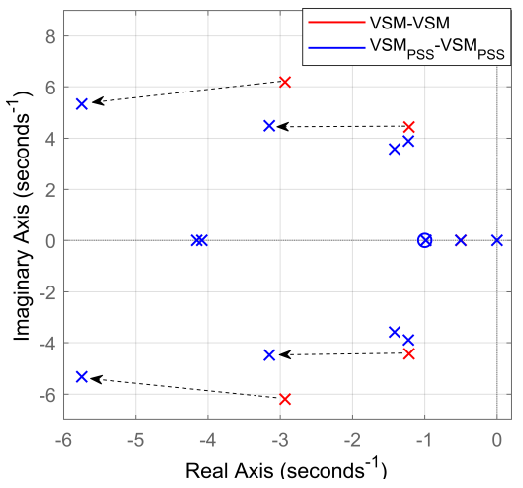

Fig. 6. Impact of PSS on LFO in an all-VSM grid

modes are well damped. Although the PSS can improve the stability of an all-VSM grid, it is not a requirement as is the case for SG dominated grids. It is noted that, as with all control designs, the above conclusion will not be valid for a poorly tuned VSM.

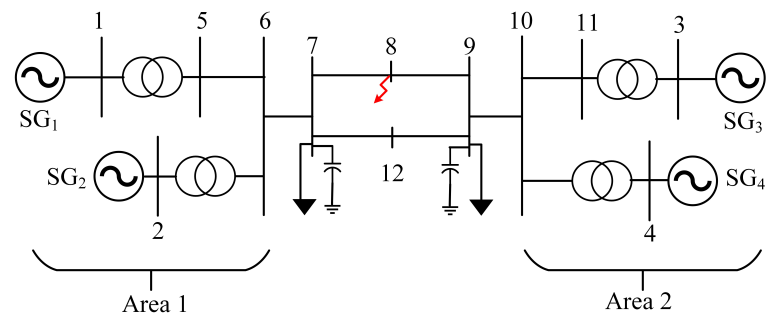

Fig. 7. IEEE benchmark two-area four-machine system [28]

\section{TRANSIENT STABILITY}

The objective of this section is to observe the dynamics of the system when subjected to large disturbances. To achieve this, the IEEE benchmark two-area four-machine system (also known as Kundur model) has been implemented (Fig. 7). This system (Fig. 7) is the dedicated IEEE benchmark for investigating LFOs and the role of PSS in power systems [60]. The full-order MATLAB/SIMULINK model of the SG with AVR and governor has been employed here. The SG and network parameters are detailed in [28], while the VSM parameters are detailed in Table. I. In Figs. 8-21, $\Delta \delta_{12}, \Delta \delta_{13}$ and $\Delta \delta_{14}$ represent the relative angular oscillation of $\mathrm{SG}_{2}$ (or 
$\mathrm{VSM}_{2}$ ), $\mathrm{SG}_{3}$ (or $\mathrm{VSM}_{3}$ ) and $\mathrm{SG}_{4}$ (or $\mathrm{VSM}_{4}$ ) with respect to $\mathrm{SG}_{1}$ (or $\mathrm{VSM}_{1}$ ). $P_{1}, P_{2}, P_{3}$ and $P_{4}$ represent the active power generated from $\mathrm{SG}_{1}$ (or $\mathrm{VSM}_{1}$ ), $\mathrm{SG}_{2}$ (or $\mathrm{VSM}_{2}$ ), $\mathrm{SG}_{3}$ (or $\mathrm{VSM}_{3}$ ) and $\mathrm{SG}_{4}$ (or $\mathrm{VSM}_{4}$ ) respectively. $Q_{1}, Q_{2}, Q_{3}$ and $Q_{4}$ represent the reactive power generated from $\mathrm{SG}_{1}$ (or $\mathrm{VSM}_{1}$ ), $\mathrm{SG}_{2}$ (or $\left.\mathrm{VSM}_{2}\right), \mathrm{SG}_{3}$ (or $\mathrm{VSM}_{3}$ ) and $\mathrm{SG}_{4}\left(\right.$ or $\mathrm{VSM}_{4}$ ) respectively. The $V$ and $f$ are measured at bus 7 (see Fig. 7). Three test cases have been investigated:

(A) Impact of VSM replacing SG.

(B) Robustness of the VSMPSS.

(C) Evaluation of an all-VSM with respect to an all-SG grid.

\section{A. Impact of VSM replacing $S G$}

To observe the transient stability of the system, a 3-phase fault is applied on the tie-line at bus 8 (see Fig. 7) at $t$ $=20 \mathrm{~s}$ for a period of $200 \mathrm{~ms}$. Four system configurations are observed. Configuration 1: four SGs with no PSS (4SG). Configuration 2: $\mathrm{SG}_{2}$ and $\mathrm{SG}_{4}$ replaced by VSMs (2SG2VSM)). Configuration 3: PSS added to the two SGs in configuration $2\left(2 \mathrm{SG}_{\mathrm{pss}}-2 \mathrm{VSM}\right)$. Configuration 4: PSS added to the two VSMs in configuration 2 (2SG-2VSM $\left.\mathrm{Vss}_{\mathrm{ps}}\right)$. The standard MB-PSS available on MATLAB/SIMULINK which offers the best performance is implemented on the SGs, while the PSS designed in section III is implemented on the VSMs. This offers a good base-line evaluation on the efficacy of the PSS employed on the VSM. The results of these tests are shown in Figs. 8-11, which are discussed as follows:

Angle stability: From Fig. 8, it is observed that the oscillation is an inter-area mode i.e. $\omega_{o s c} \approx 4.2 \mathrm{rads}^{-1}$, with machines in Area 1 swinging against machines in Area 2; $\delta_{13}$ and $\delta_{14}$ are much larger than $\delta_{12}$. For configuration 1, the system loses synchronization as the amplitude of the angular oscillations progressively increases after fault, indicating lack of sufficient damping torque. For configuration 2 , it is observed that the system is stable but poorly damped; as the angular oscillations do not increase post-fault, but will take a long period of time to be damped out. For configuration 3 , due to the addition of the MB-PSS on $\mathrm{SG}_{1}$ and $\mathrm{SG}_{3}$, the oscillation is rapidly damped and the system maintains synchronism. For configuration 4 , with the addition of PSS to the VSMs, the system oscillations are promptly damped out. It is observed that the performance of the PSS on the VSM (See Fig. 1), is very comparable to the MB-PSS on the SG. Hence, a complex PSS design is not required on the VSM to obtain satisfactory performance.

Active power oscillation: For configuration 1 (see Fig. 9), it is observed that the power oscillations progressively increases post-fault as the system is unstable. For configuration 2, the power oscillations do not increase but will take long time to be damped out. It is also observed that the inertial response from the SG is more dominant, ensuring quicker power recovery from the SG post-fault. Due to the surge in power from the SGs $\left(P_{1}\right.$ and $\left.P_{3}\right)$, the VSMs $\left(P_{2}\right.$ and $\left.P_{4}\right)$ seems to recover slowly to maintain power balance in the system. For configuration 3 , the power oscillation is promptly damped and the system maintains stability. For configuration 4 , the power oscillation is also effectively damped and it is observed that the active power dip is less than all other scenarios. This is a result of
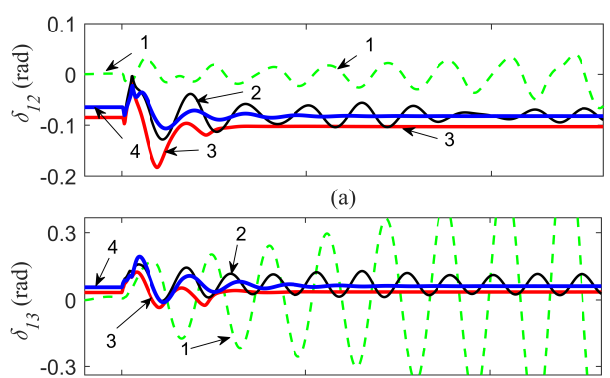

(b)

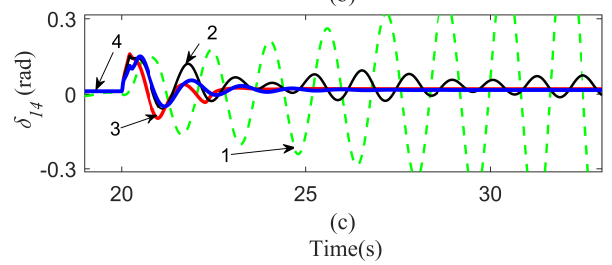

Fig. 8. Rotor angle: $1-4 S G, 2-2 S G-2 V S M, 3-2 S_{\mathrm{pss}}-2 V S M$, 4-2SG-2VSM pss.

the increased $Q$ injection due to PSS action, which leads to a corresponding reduction in voltage sag (see Fig. 11(a)).

Reactive power injection: It is observed (see Fig. 10) that, the $Q$ injected in configuration 1 and configuration 2 are comparable, though $Q$ is slightly less in configuration 1 . In configuration 3 , it is observed that the increased $Q$ injection on $\mathrm{SG}_{1}$ and $\mathrm{SG}_{3}$ is accompanied by transient absorption of $Q$ in the VSMs $\left(Q_{2}\right.$ and $\left.Q_{4}\right)$ to maintain $Q$ balance in the system. Similarly, in configuration 4 the SGs $\left(Q_{1}\right.$ and $\left.Q_{3}\right)$ transiently absorb $Q$ due to the increased $Q$ injection on the VSMs employing PSS. In comparison with the other scenarios, configuration 4 injects the maximum $Q$ during fault, thus ensuring the best voltage support.

Voltage: It is observed (see Fig. 11(a)) that the voltage dip in configurations 2 and 3 are similar. The voltage dip in configuration 1 is the largest because it injects the least $Q$ during fault. On the contrary, the least voltage dip is observed in configuration 4 due to the maximum $Q$ injected. This ensures a better voltage support in configuration 4 than all other configurations. The post-fault voltage swell and dip observed on configuration 3 and configuration 4 respectively, are due to the stabilizing signals injected into the system by the PSS.

Frequency: It is observed (see Fig. 11(b)), that configuration 1 exhibits the least deviation during fault. For configurations $2-4$, the frequency is maintained within nominal value $\pm 1 \%$ [55], and is not impacted by the PSS. From this test, it is observed that the replacement of SGs with VSM improves damping of inter-area LFOs. However, PSS is required for satisfactory performance.

\section{B. VSM PSS Robustness test}

To evaluate the robustness of the $\operatorname{VSM}_{\mathrm{PSS}}$ (see Fig. 1), the following scenarios are simulated on the same SG (or VSM) configuration as sub-section IV-A: (1) local fault, (2) fault on a heavily loaded tie-line, and (3) reversal of power flow on heavily loaded tie-line: 

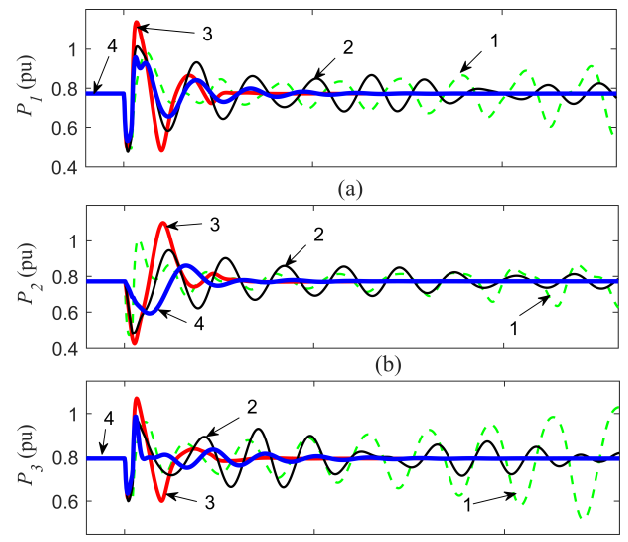

(c)

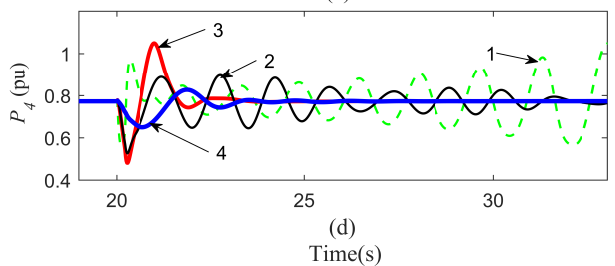

Fig. 9. Active Power (pu):1-4SG, 2-2SG-2VSM, 3-2SG $\mathrm{pss}_{\mathrm{s}}-2 \mathrm{VSM}$, 4-2SG-2VSM

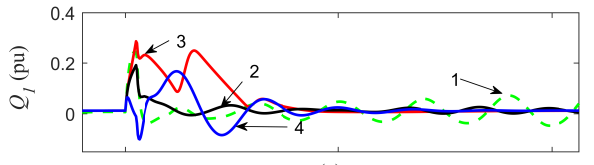

(a)
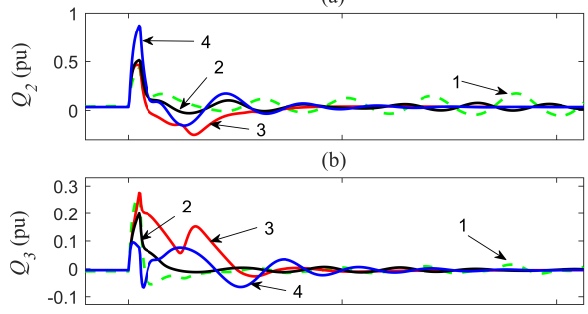

(c)

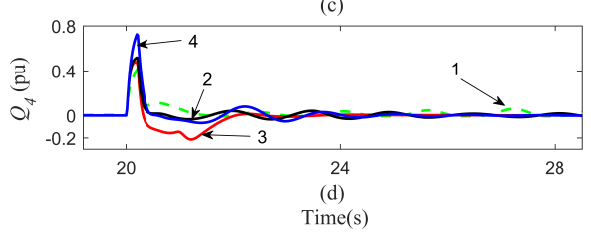

Fig. 10. Reactive Power (pu): 1-4SG, 2-2SG-2VSM, 3-2SG $\mathrm{pss}-2 \mathrm{VSM}$, 4-2SG-2VSM pss $_{\text {. }}$
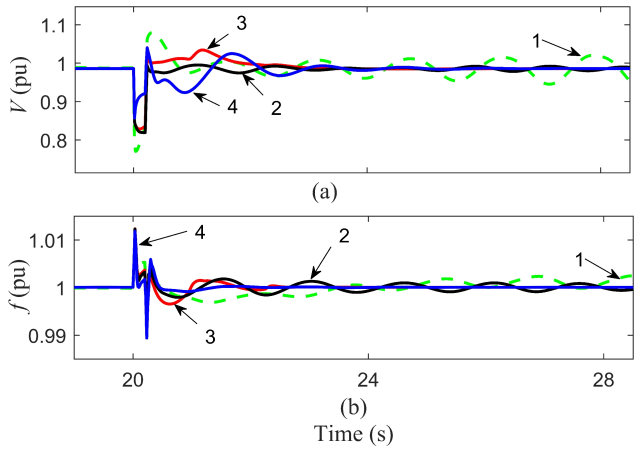

Fig. 11. (a) Voltage (pu): 1-4SG, 2-2SG-2VSM, 3-2SG $\mathrm{pss}_{\mathrm{s}}-2 \mathrm{VSM}$,

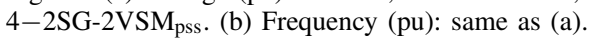

1) Local Fault: To observe the transient stability in the event of a local fault, a 3-phase fault is applied at bus 6 (see Fig. 7) at $t=20 \mathrm{~s}$ for a period of $200 \mathrm{~ms}$. The results of this tests are shown in Figs. $12 \& 13$.

Angle stability: From Fig. 12, it is observed that the local fault is more severe than the fault applied at the tie-line (see Fig. 8), due to the proximity of the fault to the SG (or VSM). For configuration 1, the system instantaneously falls out-ofstep post-fault. For configuration 2, the system is marginally stable, as the angular oscillations are not incremental postfault. For configurations 3 and 4 , the oscillation is rapidly damped due to the PSS action on the SG and VSM respectively.

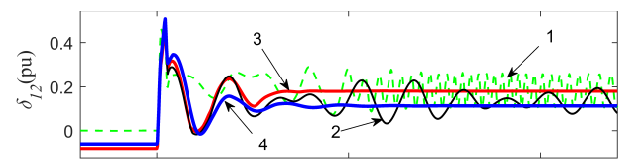

(a)

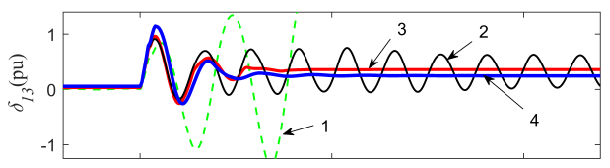

(b)

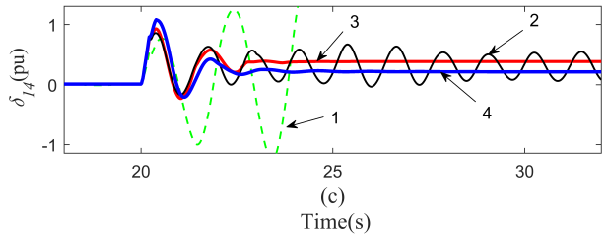

Fig. 12. Rotor angle: 1-4SG, 2-2SG-2VSM, 3-2SG pss $-2 \mathrm{VSM}$, 4-2SG-2VSM $\mathrm{pss}$.

Active Power oscillation: The power oscillation (see Fig. 13) for configurations 1 and 2, will trigger protective relays i.e outof-step and pole slip protection relays [30]. For configurations 3 and 4, the oscillations are promptly damped, and the performance of the $\mathrm{VSM}_{\mathrm{PSS}}$ (configuration 4) is comparable with MB-PSS (configuration 3) applied on the SG.

2) Fault on a heavily loaded tie-line: The load at area 2 (see Fig. 7) is increased from $2 \mathrm{pu}$ to $2.4 \mathrm{pu}$, leading to an increase in the tie-line power flow from $413 \mathrm{MW}$ to $645 \mathrm{MW}$. The fault is applied at the tie-line at bus 8 (same as sub-section IV-A). The test results are shown in Figs. $14 \& 15$.

Angle stability: From Fig. 14, it is observed that the angular oscillations are undamped and poorly damped for configurations 1 and 2 respectively. For configuration 3 and 4, the oscillations are promptly damped.

Active Power oscillation: From Fig. 15, it is observed that the power oscillations are unsatisfactory for configurations 1 and 2. The result for configurations 3 and 4 illustrate that the oscillations are promptly damped post-fault, and demonstrate the efficacy of the PSS on the VSM.

3) Reversal of power flow on heavily loaded tie-line: Here, the loads at Area 1 and Area 2 are swapped (see Fig. 7). Thereafter, the load at Area 1 is increased from 2 pu to 2.4 $\mathrm{pu}$, resulting in a net export of $645 \mathrm{MW}$ power from Area 2 to Area 1. The fault is applied at the tie-line at bus 8 (same 


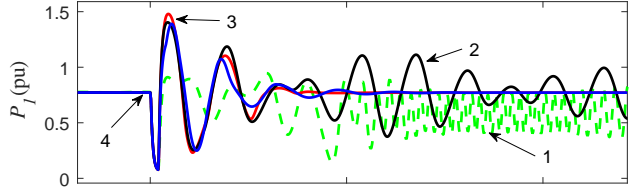

(a)

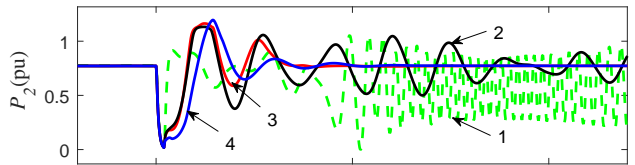

(b)

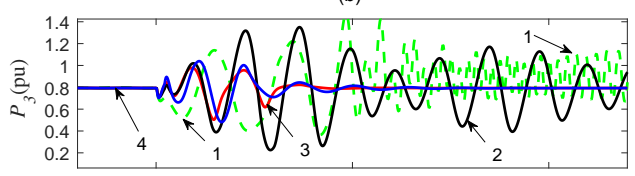

(c)

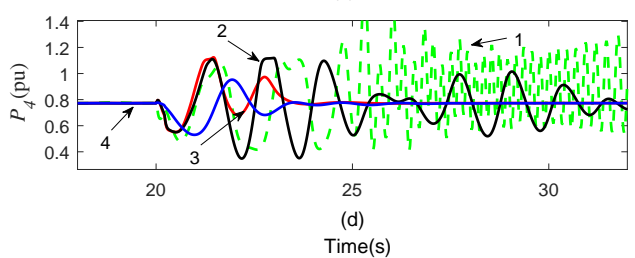

Fig. 13. Active Power (pu):1-4SG, 2-2SG-2VSM, 3-2SG $\mathrm{pss}_{\mathrm{p}}-2 \mathrm{VSM}$, 4-2SG-2VSM
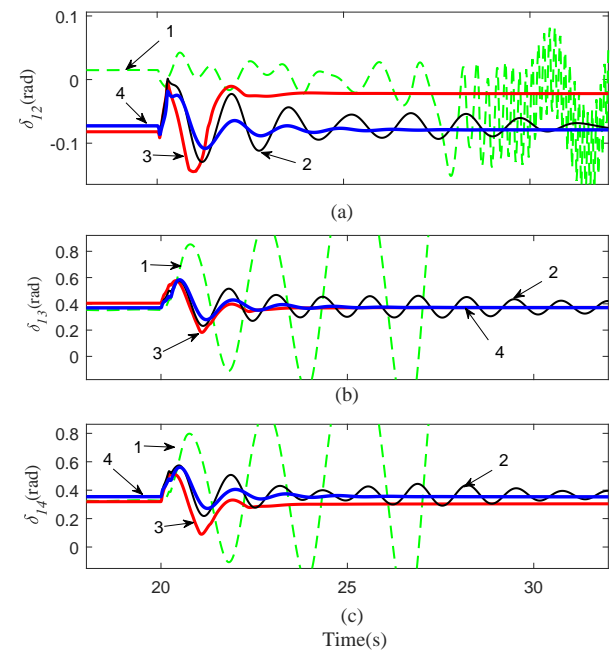

Fig. 14. Rotor angle: 1-4SG, 2-2SG-2VSM, 3-2SG $\mathrm{pss}_{\mathrm{s}}-2 \mathrm{VSM}$, 4-2SG-2VSM $\mathrm{pss}_{\text {. }}$.

as sub-section IV-A). The test results are shown in Figs. $16 \&$ 17.

Angle stability: The results in Figs. $16 \&$ 17, illustrate that the system performance is unsatisfactory for both configurations 1 and 2. For configuration 3 and 4 (see Fig. 16), the oscillations are promptly damped. Unlike [5], the VSMPSS performance is not impacted by the reversal of the tie-line power flow.

Active Power oscillation: From Fig. 17, it is observed that the system performance is satisfactory for configurations 3 and 4.

The above tests show that the $\mathrm{VSM}_{\mathrm{PSS}}$ is robust, as it

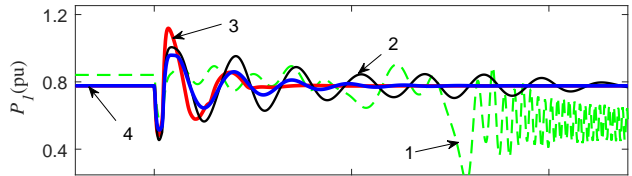

(a)

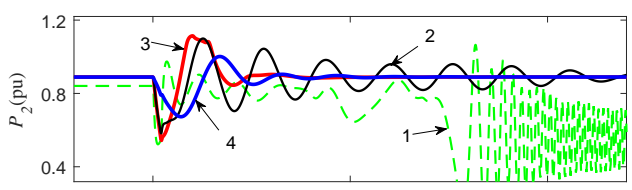

(b)
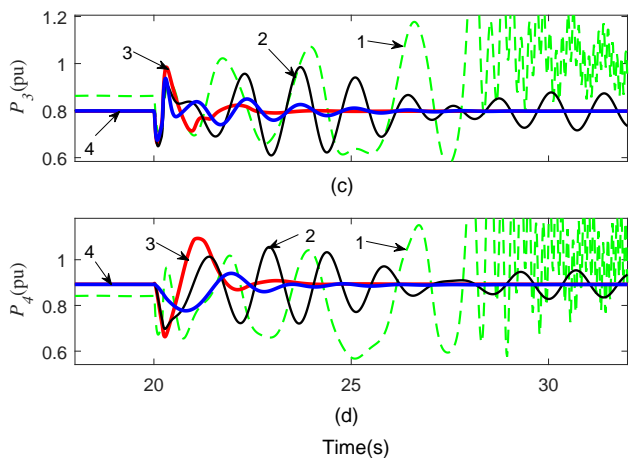

Fig. 15. Active Power (pu):1-4SG, 2-2SG-2VSM, 3-2SG $\mathrm{pss}-2 \mathrm{VSM}$, 4-2SG-2VSM $\mathrm{Vss}_{\text {. }}$

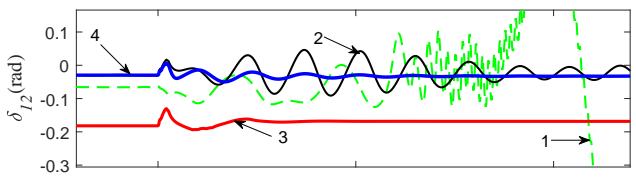

(a)

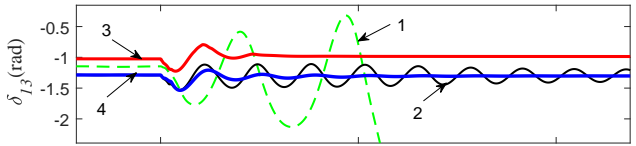

(b)

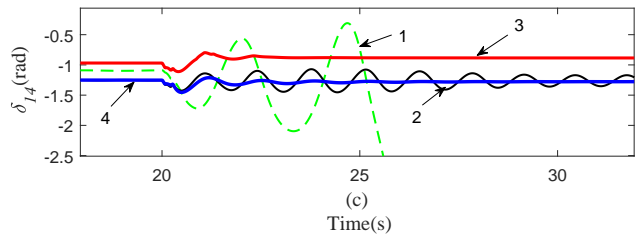

Fig. 16. Rotor angle: 1-4SG, 2-2SG-2VSM, 3-2SG $\mathrm{pss}-2 \mathrm{VSM}$, 4-2SG-2VSM $\mathrm{pss}_{\text {. }}$

provides adequate damping and satisfactory performance for various severe fault scenarios. The results also demonstrate that complex PSS designs (e.g. MB-PSS) are not required for satisfactory operation.

\section{Evaluation of an all-VSM with respect to an all-SG grid}

The objective is to observe the overall performance of an all-SG system in comparison to an all-VSM system. The same fault scenario employed in sub-section IV-A is applied here. Three system configurations are considered here. Configuration 1: four SGs no PSS (4SG). Configuration 2: four SGs with four MB-PSSs $\left(4 \mathrm{SG}_{\mathrm{pss}}\right)$. Configuration 3: four VSMs no PSS 


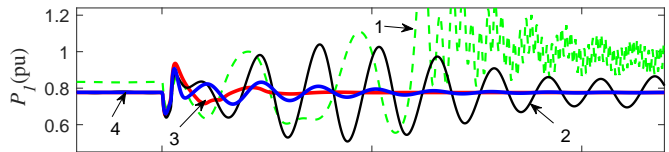

(a)

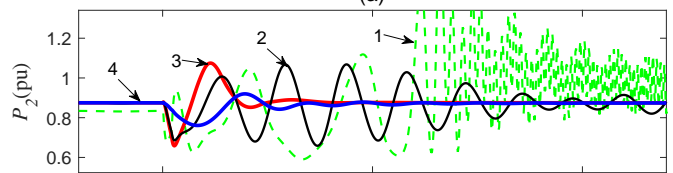

(b)

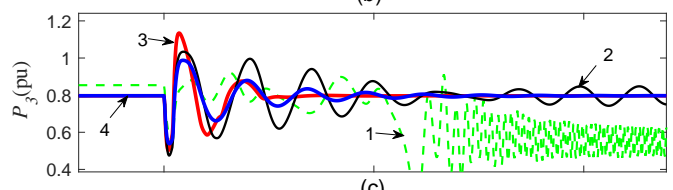

(c)

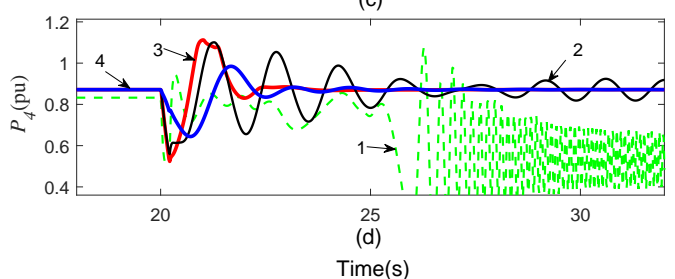

Fig. 17. Active Power (pu):1-4SG, 2-2SG-2VSM, 3-2SG pss -2 VSM, 4-2SG-2VSM pss. .

(4VSM). Configuration 1 is same as the configuration 1 in subsection IV-A, and is illustrated for the purpose of comparison and is not further discussed. The results are illustrated in Figs. 18-21.

Angle Stability: For configuration 2 (see Fig. 18), the rotor angle oscillation is rapidly damped post-fault due to the MBPSSs action. For configuration 3, no oscillation is observed in the system post-fault, however there is a net angular displacement post-fault. This is because $\delta$ is determined by PLL in the VSM, and is not required to return to pre-fault values to maintain stability, while $\delta$ is a function of the swing equation in the SG, which ensures $\delta$ returns to pre-fault values to maintain stability.

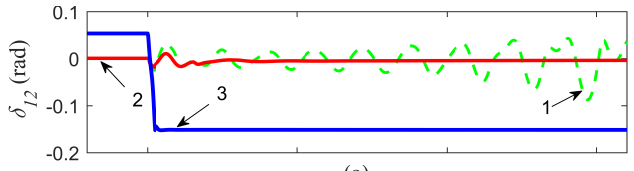

(a)

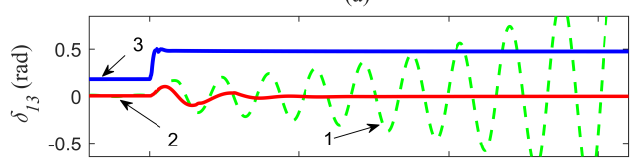

(b)

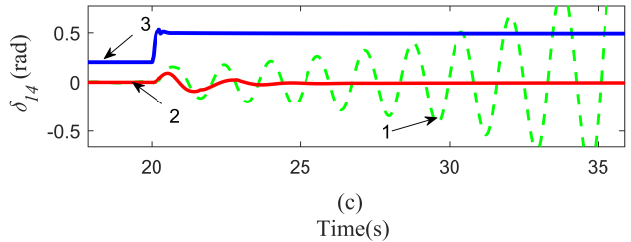

Fig. 18. Rotor angle: $1-4 \mathrm{SG}, 2-4 \mathrm{SG}_{\mathrm{pss}}, 3-4 \mathrm{VSM}$.

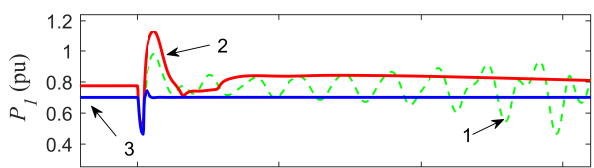

(a)

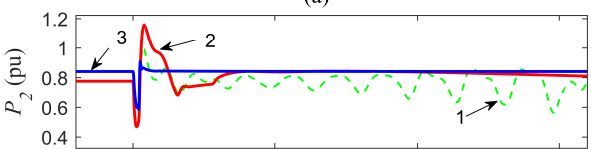

(b)

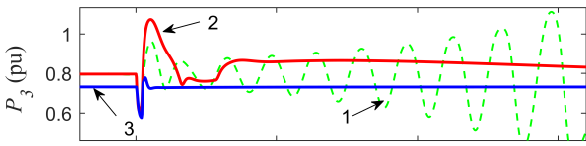

(c)

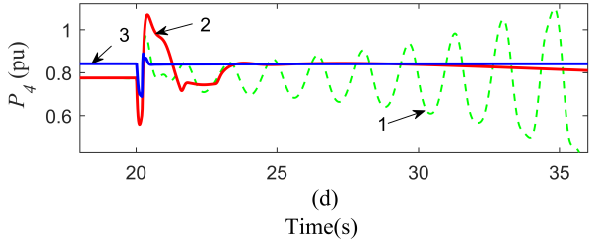

Fig. 19. Power (pu): 1-4SG, 2-4SG $\mathrm{pss}_{\mathrm{ps}}, 3-4 \mathrm{VSM}$.

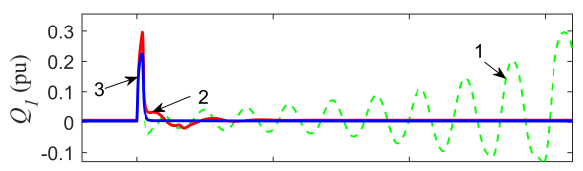

(a)

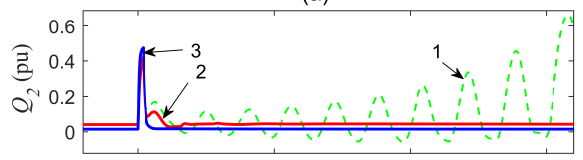

(b)

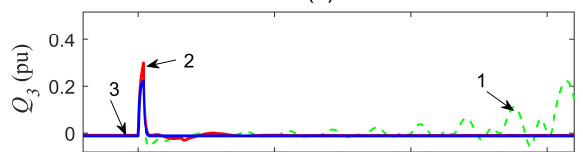

(c)

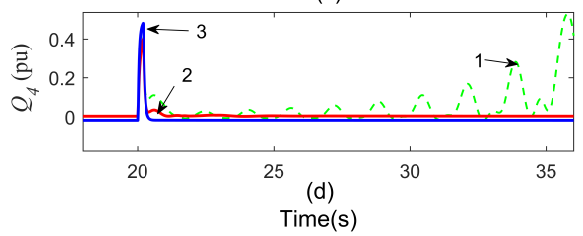

Fig. 20. Reactive Power (pu): 1-4SG, 2-4SG $\mathrm{pss}_{\text {, }}$ 3-4VSM.

Active Power oscillation: As shown in configuration 3 of Fig. 19, since no (inter-area) oscillations are generated in an all-VSM system, PSS is not required. Recovery time of $P$ in configuration 2 and configuration 3 (see Fig. 19) are similar, however there is less overshoot on configuration 3 , and the system recovers to pre-fault operating condition much faster.

Reactive Power injection: Similar response is observed (see Fig. 20) on both configuration 2 and 3 with respect to the reactive power injected during fault. This shows that the VSM can adequately support the grid voltage when SGs are relinquished.

Voltage: As shown in Fig. 21(a), post-fault voltage regulation on configuration 3 is much better than that of con- 


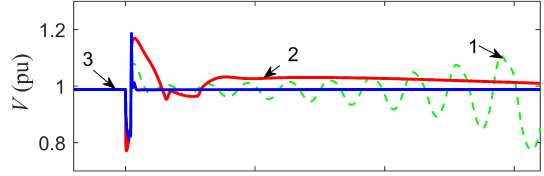

(a)

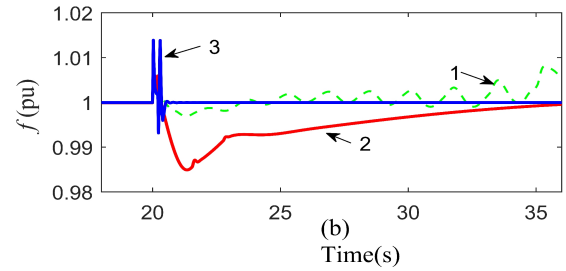

Fig. 21. (a) Voltage (pu): 1-4SG, 2-4SG $\mathrm{pss}, 3-4 \mathrm{VSM}$. (b) Frequency (pu): same as (a).

figuration 2. The voltage dip during fault is similar and the discrepancies observed may be attributed to the differences in equivalent impedance the SG and VSM present to the system, since injected $Q$ is similar on both VSM and SG.

Frequency: Frequency regulation (see Fig. 21(b)) seems better in configuration 3 than configuration 2 , as the frequency returns to pre-fault condition much quicker. It is also observed that the employment of PSS on the SG has negative impact on the frequency nadir of the system.

From this test case, it is observed that for an all-VSM grid, inter-area oscillations do not exist, hence PSS is not required. This corroborates the observation from the smallsignal analysis, which illustrates that the LFO modes for allVSM grids are well damped and the PSS is redundant. Based on these results, one may suggest decoupling all SGs from the network using VSM-controlled AC-DC-AC converter (a similar structure proposed in [22] for wind turbines). This will eliminate LFOs, smoothen transition to a sustainable all-VSM system and enhance system stability.

\section{CONCLUSION}

This paper investigated the impact of VSM based RESs on the LFOs in the power system. This was achieved using a detailed two-machine state space model to analyze the smallsignal stability, while the transient stability was investigated in an IEEE bench mark dedicated for inter-area oscillations. From the small-signal stability analysis, it was observed that when VSM replaces SG, an additional LFO is added to the system. This LFO is sufficiently damped and does not adversely impact the system stability. The PLL and virtual governor from the VSM play a major role on the LFO interaction with the SG. The net impact of the SG replacement with VSM is an improved damping of the LFO modes. However, the VSM must be integrated with PSS for satisfactory performance when interconnected with SGs. The robustness of the VSM PSS $_{\text {Pas }}$ validated for different test scenarios. For an all-VSM system, the LFO modes are well damped and inter-area oscillations do not exist, which makes using a PSS redundant. Thus, to eliminate LFOs without the need for a PSS, it is pertinent to decouple the SGs from the grid using VSMs. The authors recognize that, due to the wide variant VSM algorithms in literature, the conclusions here may not be consistent for all topologies, and will not be valid for a poorly designed VSM. The results of the transient analysis closely match the small-signal analysis, which shows that the two-machine test bed accurately describes the dynamics of the participating generators and can be employed for investigating the LFOs in VSMs with diverse dynamics.

\section{REFERENCES}

[1] M. Edrah, K. L. Lo, and O. Anaya-Lara, "Impacts of high penetration of DFIG wind turbines on rotor angle stability of power systems," IEEE Transactions on Sustainable Energy, vol. 6, no. 3, pp. 759-766, Jul. 2015.

[2] J. Ying, X. Yuan, J. Hu, and W. He, "Impact of inertia control of DFIG-Based WT on Electromechanical Oscillation Damping of SG," IEEE Transactions on Power Systems, vol. 33, no. 3, pp. 3450-3459, May 2018.

[3] M. Garmroodi, D. J. Hill, G. Verbič, and J. Ma, "Impact of tie-line power on inter-area modes with increased penetration of wind power," IEEE Transactions on Power Systems, vol. 31, no. 4, pp. 3051-3059, Jul. 2016.

[4] L. Huang, H. Xin, and Z. Wang, "Damping low-frequency oscillations through VSC-HVdc stations operated as virtual synchronous machines," IEEE Transactions on Power Electronics, vol. 34, no. 6, pp. 5803-5818, Jun. 2019.

[5] J. Zuo, Y. Li, D. Shi, and X. Duan, "Simultaneous Robust Coordinated Damping Control of Power System Stabilizers (PSSs), Static Var Compensator (SVC) and Doubly-Fed Induction Generator Power Oscillation Dampers (DFIG PODs) in Multimachine Power Systems," Energies, vol. 10, no. 4, 2017.

[6] National Grid, Voltage and Frequency Dependency. National Grid, 2018, [Accessed on 19-Feb-2020]. [Online]. Available: https : / / www . nationalgrid . com/sites / default/ files / documents / SOFReport FrequencyandVoltageassessment.pdf.

[7] R. Smith, System Operability Framework. National Grid, 2015 [Accessed on 19-Feb-2020]. [Online]. Available: https : / / www . nationalgrideso.com/document/63461/download.

[8] F. Slye, Future Energy Scenarios. National Grid, 2019, [Accessed on 19-Feb-2020]. [Online]. Available: http://fes.nationalgrid.com/media/ 1409/fes-2019.pdf.

[9] T. Shintai, Y. Miura, and T. Ise, "Oscillation damping of a distributed generator using a virtual synchronous generator," IEEE Transactions on Power Delivery, vol. 29, no. 2, pp. 668-676, Apr. 2014.

[10] J. Alipoor, Y. Miura, and T. Ise, "Power system stabilization using virtual synchronous generator with alternating moment of inertia," IEEE Journal of Emerging and Selected Topics in Power Electronics, vol. 3, no. 2, pp. 451-458, Jun. 2015.

[11] W. Du, Q. Fu, and H. F. Wang, "Power system small-signal angular stability affected by virtual synchronous generators," IEEE Transactions on Power Systems, vol. 34, no. 4, pp. 3209-3219, 2019.

[12] K. Shi, C. Chen, Y. Sun, P. Xu, Y. Yang, and F. Blaabjerg, "Rotor inertia adaptive control and inertia matching strategy based on parallel virtual synchronous generators system," IET Generation, Transmission Distribution, vol. 14, no. 10, pp. 1854-1861, 2020.

[13] C. Sun, G. Joos, and F. Bouffard, "Identification of low-frequency oscillation mode and improved damping design for virtual synchronous machines in microgrid," IET Generation, Transmission Distribution, vol. 13, no. 14, pp. 2993-3001, 2019.

[14] J. R. Pérez, J. A. Suul, S. D’Arco, A. Rodríguez-Cabero, and M. Prodanovic, "Virtual synchronous machine control of VSC-HVDC for power system oscillation damping," in IECON 2018 - 44th Annual Conference of the IEEE Industrial Electronics Society, Oct. 2018 , pp. 6026-6031.

[15] W. Sheng, Y. Wang, B. Liu, S. Duan, and M. Wu, "Virtual synchronous generator strategy for suppressing output power fluctuation without additional energy storage," IET Power Electronics, vol. 13, no. 3, pp. 602-610, 2020.

[16] G. W. Moshe Blau, "Synchronverters used for damping inter-area oscillations in two-area power systems," in International Conference on Renewable Energies and Power Quality (ICREPQ), Mar. 2018.

[17] Y. Li, L. Fan, and Z. Miao, "Wind in Weak Grids: Low-Frequency Oscillations, Subsynchronous Oscillations, and Torsional Interactions," IEEE Transactions on Power Systems, vol. 35, no. 1, pp. 109-118, 2020 
[18] L. Fan, "Modeling Type-4 Wind in Weak Grids," IEEE Transactions on Sustainable Energy, vol. 10, no. 2, pp. 853-864, 2019.

[19] L. Fan and Z. Miao, "An explanation of oscillations due to wind power plants weak grid interconnection," IEEE Transactions on Sustainable Energy, vol. 9, no. 1, pp. 488-490, Jan. 2018.

[20] J. Quintero, V. Vittal, G. T. Heydt, and H. Zhang, "The impact of increased penetration of converter control-based generators on power system modes of oscillation," IEEE Transactions on Power Systems, vol. 29 , no. 5, pp. 2248-2256, 2014.

[21] S. Li, "Low-frequency oscillations of wind power systems caused by doubly-fed induction generators," Renewable Energy, vol. 104, pp. 129-138, 2017, ISSN: 0960-1481.

[22] B. Muftau, M. Fazeli, and A. Egwebe, "Stability analysis of a PMSG based Virtual Synchronous Machine," Electric Power Systems Research, vol. 180, p. 106170 , Mar. 2020.

[23] D. Trudnowski, D. Kosterev, and J. Undrill, "PDCI damping control analysis for the western North American power system," in 2013 IEEE Power Energy Society General Meeting, 2013, pp. 1-5.

[24] F. Wilches-Bernal, R. H. Byrne, and J. Lian, "Damping of Inter-Area Oscillations via Modulation of Aggregated Loads," IEEE Transactions on Power Systems, vol. 35, no. 3, pp. 2024-2036, 2020.

[25] J. Lian, S. Wang, and M. E. J. Hansen, Universal wide-area damping control for mitigating interarea oscillations in power systems. Pacific Northwest National Laboratory, Richland, WA, USA, Tech. Rep. PNNL-27351, 2017.

[26] C. Zhang, D. Ke, Y. Sun, C. Y. Chung, and J. Xu, "Investigations of Large-Scale Voltage-Dependent Loads for Damping Inter-Area Oscillations: Mechanism and Robust Decentralized Control," IEEE Transactions on Power Systems, vol. 33, no. 6, pp. 6037-6048, 2018.

[27] J. Lian, Q. Zhang, L. D. Marinovici, R. Fan, and J. Hansen, "WideArea Demand-Side Control for Inter-Area Oscillation Mitigation in Power Systems," in 2018 IEEE/PES Transmission and Distribution Conference and Exposition (T D), 2018, pp. 1-5.

[28] P. Kundur, N. Balu, and M. Lauby, Power System Stability and Control. McGraw-Hill Education, 1994.

[29] P. M. Anderson and A. A. Fouad, Power System Control and Stability. IEEE, 2003.

[30] J. Machowski, Z. Lubosny, J. Bialek, and J. Bumby, Power System Dynamics: Stability and Control. Wiley, 2020.

[31] Y. Chi, B. Tang, J. Hu, X. Tian, H. Tang, Y. Li, S. Sun, L. Shi, and L. Shuai, "Overview of mechanism and mitigation measures on multi-frequency oscillation caused by large-scale integration of wind power," CSEE Journal of Power and Energy Systems, vol. 5, no. 4, pp. 433-443, 2019.

[32] W. Du, J. Bi, and H. F. Wang, "Small-signal angular stability of power system as affected by grid-connected variable speed wind generatorsa survey of recent representative works," CSEE Journal of Power and Energy Systems, vol. 3, no. 3, pp. 223-231, Sep. 2017.

[33] T. Sadamoto, A. Chakrabortty, T. Ishizaki, and J. Imura, "Retrofit control of wind-integrated power systems," IEEE Transactions on Power Systems, vol. 33, no. 3, pp. 2804-2815, May 2018.

[34] J. Ma, Y. Qiu, Y. Li, W. Zhang, Z. Song, and J. S. Thorp, "Research on the Impact of DFIG Virtual Inertia Control on Power System Small-Signal Stability Considering the Phase-Locked Loop," IEEE Transactions on Power Systems, vol. 32, no. 3, pp. 2094-2105, May 2017.

[35] Y. Wang and Y. Yuan, "Inertia provision and small signal stability analysis of a wind-power generation system using phase-locked synchronized equation," Sustainability, vol. 11, no. 5, 2019.

[36] Z. Liu, C. Liu, G. Li, Y. Liu, and Y. Liu, "Impact Study of PMSGBased Wind Power Penetration on Power System Transient Stability Using EEAC Theory," Energies, vol. 8, no. 12, pp. 13419-13441, 2015.

[37] T. Surinkaew and I. Ngamroo, "Coordinated Robust Control of DFIG Wind Turbine and PSS for Stabilization of Power Oscillations Considering System Uncertainties," IEEE Transactions on Sustainable Energy, vol. 5, no. 3, pp. 823-833, 2014.

[38] T. Surinkaew and I. Ngamroo, "Hierarchical Coordinated Wide Area and Local Controls of DFIG Wind Turbine and PSS for Robust Power Oscillation Damping," IEEE Transactions on Sustainable Energy, vol. 7, no. 3, pp. 943-955, 2016.

[39] M. J. Morshed and A. Fekih, "A Probabilistic Robust Coordinated Approach to Stabilize Power Oscillations in DFIG-Based Power Systems," IEEE Transactions on Industrial Informatics, vol. 15, no. 10, pp. 5599-5612, 2019.

[40] M. Singh, A. J. Allen, E. Muljadi, V. Gevorgian, Y. Zhang, and S. Santoso, "Interarea oscillation damping controls for wind power plants," IEEE Transactions on Sustainable Energy, vol. 6, no. 3, pp. 967-975, 2015.

[41] E. Vittal, M. O'Malley, and A. Keane, "Rotor angle stability with high penetrations of wind generation," IEEE Transactions on Power Systems, vol. 27, no. 1, pp. 353-362, 2012.

[42] Y. Chen, R. Hesse, D. Turschner, and H. Beck, "Improving the grid power quality using virtual synchronous machines," in 2011 International Conference on Power Engineering, Energy and Electrical Drives, May 2011, pp. 1-6.

[43] Q. Zhong and G. Weiss, "Synchronverters: Inverters that mimic synchronous generators," IEEE Transactions on Industrial Electronics, vol. 58, no. 4, pp. 1259-1267, Apr. 2011.

[44] U. Tamrakar, D. Shrestha, M. Maharjan, B. P. Bhattarai, T. M Hansen, and R. Tonkoski, "Virtual inertia: Current trends and future directions," Applied Sciences, vol. 7, no. 7, 2017, ISSN: 2076-3417. DOI: 10.3390/app7070654. [Online]. Available: https://www.mdpi. com/2076-3417/7/7/654.

[45] M. Ashabani and J. Jung, "Synchronous voltage controllers: Voltagebased emulation of synchronous machines for the integration of renewable energy sources," IEEE Access, vol. 8, pp. 49497-49508, 2020.

[46] J. Liu, Y. Miura, H. Bevrani, and T. Ise, "Enhanced virtual synchronous generator control for parallel inverters in microgrids," IEEE Transactions on Smart Grid, vol. 8, no. 5, pp. 2268-2277, 2017.

[47] M. Ashabani, Y. A. I. Mohamed, M. Mirsalim, and M. Aghashabani, "Multivariable droop control of synchronous current converters in weak grids/microgrids with decoupled dq-axes currents," IEEE Transactions on Smart Grid, vol. 6, no. 4, pp. 1610-1620, 2015.

[48] Q. Zhong and Y. Zeng, "Universal droop control of inverters with different types of output impedance," IEEE Access, vol. 4, pp. 702 712, Mar. 2016.

[49] N. Pogaku, M. Prodanovic, and T. C. Green, "Modeling, analysis and testing of autonomous operation of an inverter-based microgrid," IEEE Transactions on Power Electronics, vol. 22, no. 2, pp. 613-625, Mar. 2007.

[50] Y. Levron, J. Belikov, and D. Baimel, "A tutorial on dynamics and control of power systems with distributed and renewable energy sources based on the dq0 transformation," Applied Sciences, vol. 8 , no. 9 , Sep. 2018

[51] A. J Roscoe, M. Yu, A. Dyśko, C. Booth, R. Ierna, J. Zhu, and H. Urdal, "A vsm (virtual synchronous machine) convertor control model suitable for rms studies for resolving system operator / owner challenges," 15th Wind Integration Workshop, Nov. 2016. [Online]. Available: http://strathprints.strath.ac.uk/58053/.

[52] M. Guan, W. Pan, J. Zhang, Q. Hao, J. Cheng, and X. Zheng, "Synchronous Generator Emulation Control Strategy for Voltage Source Converter (VSC) Stations," IEEE Transactions on Power Systems, vol. 30, no. 6, pp. 3093-3101, 2015.

[53] J. Zhu, J. Hu, W. Hung, C. Wang, X. Zhang, S. Bu, Q. Li, H. Urdal, and C. D. Booth, "Synthetic inertia control strategy for doubly fed induction generator wind turbine generators using lithium-ion supercapacitors," IEEE Transactions on Energy Conversion, vol. 33 , no. 2, pp. 773-783, Jun. 2018

[54] K. S. Yuko Hirase Kazuhiro Abe and Y. Shindo, "A grid-connected inverter with virtual synchronous generator model of algebraic type," IEEJ Transactions on Power and Energy, vol. 132, pp. 371-380, 4 2012.

[55] National Grid, THE GRID CODE - issue 5, revision 44. National Grid, Jun. 2020, [Accessed on 25-Jun-2020]. [Online]. Available: https : //www.nationalgrideso.com/document/162271/download.

[56] D. Baimel, J. Belikov, J. M. Guerrero, and Y. Levron, "Dynamic modeling of networks, microgrids, and renewable sources in the dq0 reference frame: A survey," IEEE Access, vol. 5, pp. 21 323-21 335, Oct. 2017.

[57] N. Yang, Q. Liu, and J. D. McCalley, "TCSC controller design for damping interarea oscillations," IEEE Transactions on Power Systems, vol. 13, no. 4, pp. 1304-1310, Nov. 1998.

[58] M. Gibbard, P. Pourbeik, and D. Vowles, Small-signal Stability, Control and Dynamic Performance of Power Systems. University of Adelaide Press, 2015.

[59] H. Ergun, J. Dave, D. Van Hertem, and F. Geth, "Optimal power flow for ac-dc grids: Formulation, convex relaxation, linear approximation, and implementation," IEEE Transactions on Power Systems, vol. 34, no. 4, pp. 2980-2990, 2019.

[60] C. Canizares et al., "Benchmark models for the analysis and control of small-signal oscillatory dynamics in power systems," IEEE Transactions on Power Systems, vol. 32, no. 1, pp. 715-722, Jan. 2017. 